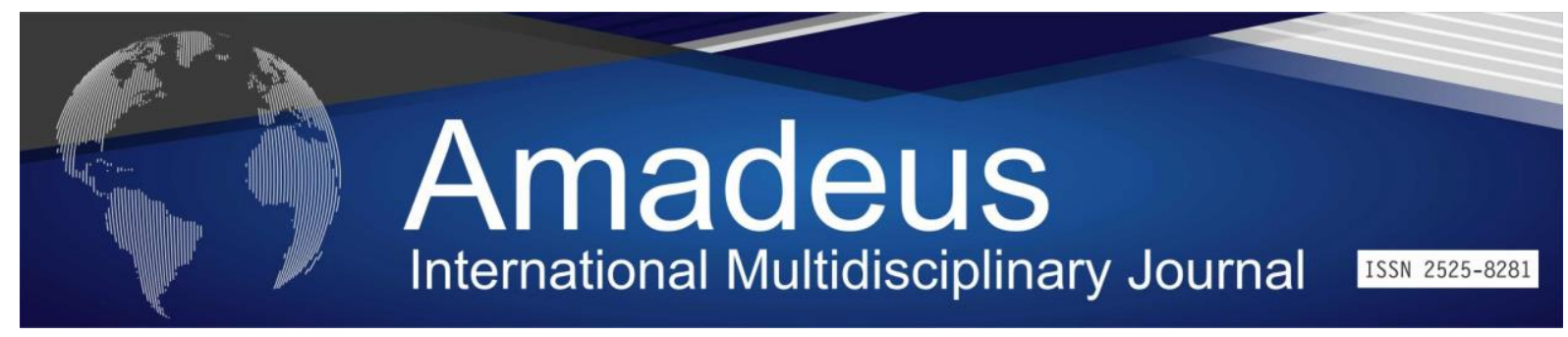

DOI: 10.14295/aimj.v5i9.122

\title{
Mental Health of the Elderly Population amid Pandemic COVID-19
}

Luiza Thayline Vieira Caldas $^{1}$

João Victor da silva Alcântara ${ }^{2}$,

Paula Pably Batista

Arraes $^{3}$

Priscila Teixeira Dias ${ }^{4}$,

Ramille Gonçalves

Oliveira $^{5}$,

Modesto Leite Rolim Neto 6 , Camila Bezerra Nobre
Abstract: With the reality of the pandemic by COVID-19 extending to alarming levels, the coexistence forced by the need for social withdrawal, We observe the difficulty of maintaining mental stability increasingly discussed. The fear of contracting the disease, as well as thinking about its consequences, makes the population anxious. In this sense, age presents itself as the major risk factor for more severe manifestations of the disease, hence, elderly people are at greater vulnerability for the development of psychological and psychiatric disorders. It is important to ask how our elderly people are living, if a pandemic scenario would really be necessary for the population in question to have visibility. Talking about the health of the elderly both mentally and physically is everyone's duty. In any circumstance, it is up to society to ensure that they can live with health, vitality and the quality of life that every human being deserves.

Keywords: COVID-19; Pandemic; Old people; Mental health.

\footnotetext{
${ }^{1}$ Acadêmica de Medicina - Faculdade de Medicina de Juazeiro do Norte Estácio de Sá - Juazeiro do Norte, Ceará, Brasil; luizathayline@ gmail.com

${ }^{2}$ Acadêmico de Medicina - Faculdade de Medicina de Juazeiro do Norte - Estácio de Sá - Juazeiro do Norte, Ceará, Brasil ; jvictoralcantara@outlook.com.br

${ }^{3}$ Acadêmica de Medicina - Faculdade de Medicina de Juazeiro do Norte - Estácio de Sá - Juazeiro do Norte, Ceará, Brasil ; arraespaula@gmail.com

${ }^{4}$ Acadêmica de Medicina - Faculdade de Medicina de Juazeiro do Norte - Estácio de Sá - Juazeiro do Norte, Ceará, Brasil ; priscila21.02@outlook.com

${ }^{5}$ Acadêmica de Medicina - Faculdade de Medicina de Juazeiro do Norte - Estácio de Sá - Juazeiro do Norte, Ceará, Brasil ; ramille.goncalves@ hotmail.com

${ }^{6}$ Pós-Doutor em Saúde Pública pela Universidade de São Paulo-USP e Professor do curso de Medicina da Estácio\Faculdade de Medicina de Juazeiro do Norte-FMJ. modestorolim@yahoo.com.br;

${ }^{7}$ Doutora em Biotecnologia pela Universidade Estadual do Ceará-UECE e Professora da Estácio\Faculdade de Medicina de Juazeiro do Norte-FMJ. milinhanobre@gmail.com.
} 


\title{
Saúde Mental da População Idosa em meio à Pandemia COVID-19
}

\begin{abstract}
Resumo: Com a realidade da pandemia pela COVID-19 se estendendo a níveis alarmantes, a convivência forçada pela necessidade de afastamento social, Observamos cada vez mais discutida a dificuldade de manter a estabilidade mental. O medo de contrair a doença, bem como pensar nas suas consequências deixam a população ansiosa. Neste sentido, a idade apresenta-se como o fator de risco maior para manifestações mais graves da doença, daí, as pessoas idosas encontram-se em maior vulnerabilidade para o desenvolvimento de transtornos psicológicos e psiquiátricos. É importante questionar como nossos idosos estão vivendo, se realmente seria necessário um cenário de pandemia para que a população em questão tivesse visibilidade. Falar sobre a saúde dos idosos tanto mental quanto física é dever de todos. Em qualquer circunstância, cabe à sociedade garantir que possam viver com saúde, vitalidade e com a qualidade de vida que todo ser humano merece.
\end{abstract}

Palavras-chave: COVID-19; Pandemia; Idoso; Saúde mental.

\section{Introdução}

No atual cenário de pandemia pela COVID-19, a dificuldade de manter a estabilidade mental se vê cada vez mais presente e discutida. A possibilidade de contrair a doença e o medo nela inserido assolam toda a população, todavia, a idade apresenta-se como o fator de risco mais bem esclarecido para a apresentação de manifestações mais graves da doença (Li et al, 2020), e, com isso, as pessoas idosas encontram-se em maior vulnerabilidade para o desenvolvimento de transtornos psicológicos e psiquiátricos determinados pela ansiedade inerente à situação (Wang et al, 2020). Além disso, as medidas de isolamento instauradas para conter a elevada transmissibilidade do vírus e diminuir o contágio, devido ao sentimento de afastamento e solidão que proporcionam, estão relacionadas ao agravo ou mesmo desenvolvimento de quadros depressivos e/ou ansiosos, além de questões orgânicas como elevado risco de morbidade cardiovascular e cerebrovascular, declínio cognitivo acelerado e redução da capacidade imune do organismo (Steinman; Perry; Perissinotto, 2020).

Um dado caminha lado a lado com a problemática em relação às mazelas psicossociais causadas na população idosa pelo novo coronavírus. Segundo o Dr. Hans Henri P Kluge, diretor regional da World Health Organization (WHO) na Europa, pesquisas apontam que 
mais de $95 \%$ das mortes registradas até o dado momento no velho continente têm como principal vítima a pessoa idosa, acima de 60 anos (WHO, 2020). Isso gera, mesmo que inconscientemente, um ambiente de pânico entre boa parte da população mais velha (O Globo, 2020), que observa o dado alarmante como uma certeza de gravidade em caso de contágio, levando a reações patológicas como uma maior frequência de pensamentos hipocondríacos e até potencialização de problemas psíquicos pré-existentes, como síndrome do pânico e ansiedade, podendo até confundir os sintomas respiratórios do COVID-19. (NYC GOV, 2020)

Há a necessidade de um olhar crítico ao assunto, tendo como foco a ajuda à pessoa mais velha em tempos de pandemia. Esse apoio deve partir de todas as pessoas, pois uma união entre todos fortaleceria essa população que sofre tanto com os efeitos da doença e da quarentena. Medo de contrair a doença da sua maneira grave e falecer, tristeza por não poder se encontrar com familiares e amigos e, por fim, ansiedade por não saber o que pode acontecer em um futuro próximo; esses são alguns dos sentimentos mais comuns aos idosos, que enfrentam diariamente uma luta pessoal para manter a sanidade mental e física em uma época tão difícil (Armitage; Nellums, 2020). Esse apoio inclui acesso seguro à alimentos, medicamentos e informações de saúde social e mental para manter o bem estar emocional dos envolvidos (WHO, 2020), bem como maneiras de evitar a completa solidão, como chamadas de vídeo. (The Conversation, 2020)

O isolamento social não é uma problemática inerente ao novo coronavírus. Nos EUA, cerca de $24 \%$ dos adultos residentes na comunidade com 65 anos ou mais, eram considerados socialmente isolados, e 43\% dos adultos com 65 anos ou mais, já se sentiam solitários (Reger; Stanley; Joiner, 2020). Solidão e isolamento social não são apenas questões sociais - eles também podem afetar a saúde física e mental de uma pessoa e o tecido das comunidades (Stephenson, 2020).O isolamento social foi associado a um risco significativamente aumentado de mortalidade precoce por todas as causas, sendo constatado pelos seguintes dados: um risco aumentado em 50\% de desenvolver demência, 29\% de doença cardíaca coronária incidente, $25 \%$ mortalidade por câncer, $59 \%$ de declínio funcional e $32 \%$ de derrame (Vox, 2020).

As estratégias implementadas para combate ao CODIV 19 são de notória efetividade para redução de novos casos, entretanto carregam consigo efeitos colaterais em potencial para o aumento da taxa de suicídio, como ansiedade, desengajamento social e questões econômicas (Steinman; Perry; Perissinotto, 2020). A diminuição das conexões sociais foi 
elencada como importante fator no aumento da taxa de suicídio entre idosos durante a epidemia de SARS em Hong Kong em 2003 (Reger; Stanley; Joiner, 2020).

Além disso, os problemas de saúde física exacerbados da população anciã podem aumentar essas taxas, uma vez que apresentam associação direta. Todas essas repercussões tornam o coronavírus uma ameaça ao mesmo tempo aterrorizante e isolante (Vox, 2020). Em meio ao cenário caótico atual, pode haver um lado positivo chamado de efeito pullingtogether, por meio dos quais indivíduos submetidos a uma experiência compartilhada podem se apoiar, fortalecendo a conexão social, demonstrado pela redução do número de suicídios após desastres nacionais, como o 11 de setembro. Os períodos de pós-pandemias podem modificar os pontos de vista a respeito de assuntos como a saúde e a mortalidade, tornando a vida mais significativa e a morte mais pavorosa (Stephenson, 2020).

Diante do exposto, faz-se necessário abordar a saúde mental e os perigos aos quais estão expostos os idosos além da pandemia em vigência. A abordagem acerca do isolamento social e como isso afeta a população idosa é apenas um ramo das inúmeras problemáticas atuais, dentre elas a solidão, o medo, a falta de alimentos ou medicamentos, principalmente naqueles que moram sozinhos ou não possuem algum vínculo familiar ou social para fazer suas compras essenciais (Armitage; Nellums, 2020).

Neste contexto, uma onda de solidariedade vem sendo levantada, principalmente pelos mais jovens, para sanar a questão levantada acima e, assim, diminuir a exposição deste grupo de risco. Ademais, o uso de ferramentas como a internet têm ajudado os mesmos a superar a falta das atividades cotidianas e laborais. A transmissão ao vivo de missa, shows, ligações por vídeo-chamada com os parentes e amigos estão se tornando cada vez mais comuns, inclusive naqueles que eram mais resistentes a entrar no meio digital, e tornam a rotina do isolamento mais leve e fluida $(\mathrm{G} 1,2020)$.

Com isso, um questionamento pertinente deve ser feito acerca de como nossos idosos estão vivendo, se realmente seria necessário um cenário de pandemia para que a população em questão tivesse visibilidade. Muitos estavam esquecidos, até mesmo pela sua família e tornaram-se alvo de preocupações apenas pela condição de grupo de risco, negligenciando outros aspectos de sua saúde. (Cesari; Proietti, 2020).

Falar sobre a saúde dos idosos tanto mental quanto física é dever de todos e em qualquer circunstância, cabe à sociedade garantir que eles não caiam no esquecimento novamente, pois todos merecem envelhecer com saúde e qualidade de vida. À partir dessas considerações conclui-se que o país demanda por políticas públicas que garantam maior 
visibilidade e atenção para com a pessoa idosa, em todas as dimensões, seja econômica de saúde e no acolhimento e prevenção a saúde mental.

\section{Referências}

Armitage, Richard; Nellums, Laura B. (2020). COVID-19 and the consequences of isolating the elderly. The Lancet Public Health, [s.1.], v. 5, n. 5, p. 256-256, maio. Elsevier BV. http://dx.doi.org/10.1016/s2468-2667(20)30061-x. Disponível em: https://www.thelancet.com/journals/lanpub/article/PIIS2468-2667(20)30061-

X/fulltext\#articleInformation. Acesso em: 11 maio 2020.

Cesari, M., Proietti, M. Medicina Geriátrica na Itália na época do COVID-19. J Nutr Health Aging 24, 459-460 (2020). Disponível em <https://doi.org/10.1007/s12603-020-1354-z. Acesso em: 05 maio 2020.

Garcia, Rafael (2020). Medo do COVID-19 se espalha entre idosos brasileiros. Disponível em: https://oglobo.globo.com/sociedade/coronavirus/medo-do-covid-19-se-espalha-entreidosos-brasileiros-24291464. Acesso em: 19 maio 2020.

G1 (2020). Missas, chamadas de vídeo e informação: idosos usam internet para contornar isolamento em Uberaba. Uberlândia, $\mathrm{Mg}, 03$ maio. Disponível em: https://g1.globo.com/mg/triangulo-mineiro/vida-em-casa/noticia/2020/05/03/missaschamadas-de-video-e-informacao-idosos-usam-internet-para-contornar-isolamento-emuberaba.ghtml Acesso em: 05 maio 2020.

Kluge, Hans Henri P. (2020). Statement - Older people are at highest risk from COVID-19, but all must act to prevent community spread. Disponível em: http://www.euro.who.int/en/health-topics/health-emergencies/coronavirus-covid19/statements/statement-older-people-are-at-highest-risk-from-covid-19,-but-all-must-act-toprevent-community-spread. Acesso em: 19 maio 2020.

Li, Heng et al. (2020). Coronavirus disease 2019 (COVID-19): current status and future perspectives. International Journal of Antimicrobial Agents, [s. l.], 29 mar. DOI 10.1016/j.ijantimicag.2020.105951. Disponível em: https://www.ncbi.nlm.nih.gov/pmc/articles/PMC7139247/. Acesso em: 11 maio 2020.

NYC health department (2020). Is It Anxiety, a Panic Attack, or COVID-19?. Disponível em: https://www1.nyc.gov/assets/doh/downloads/pdf/imm/covid-19-anxiety-panic.pdf. Acesso em: 19 maio 2020.

Reger M.A.; Stanley, I.H.; Joiner, T.E. (2020). Mortalidade por suicídio e doença de coronavírus 2019 - uma tempestade perfeita? JAMA Psychiatry. Publicado online em 10 de abril de 2020. doi: 10.1001 / jamapsychiatry.2020.1060

Sher L. (2020). Uma pandemia de doença infecciosa e aumento do risco de suicídio. Braz J Psychiatry. Disponível em < http://dx.doi.org/10.1590/1516-4446-2020-0989>. Acesso em: 19 maio 2020. 
Steinman, Michael A; Perry, Laura; Perissinotto, Carla M. (2020). Meeting the Care Needs of Older Adults Isolated at Home During the COVID-19 Pandemic. JAMA Intern Med. Published online April 16. doi:10.1001/jamainternmed.2020.1661

Stephenson, Joan. (2020). In Time of Social Distancing, Report's Call for Health Care System to Address Isolation and Loneliness Among Seniors Resonates. JAMA Health Forum, [s. l.], 17 mar. 2020. Disponível em: https://jamanetwork.com/channels/healthforum/fullarticle/2763354?resultClick=1. Acesso em: 11 maio 2020.

Todd, Joe; Atabakhsh, Victoria (2020). Video chats can ease social isolation for older adults during coronavirus pandemic. Disponível em: https://theconversation.com/video-chats-canease-social-isolation-for-older-adults-during-coronavirus-pandemic-135890. Acesso em: 19 maio 2020.

Vox (2020). Coronavirus will also cause a loneliness epidemic. [s.l.], 12 mar. Disponível em: https://www.vox.com/2020/3/12/21173938/coronavirus-covid-19-social-distancing-elderlyepidemic-isolation-quarantine. Acesso em: 05 maio 2020.

Wang, Huali et al. (2020). Coronavirus epidemic and geriatric mental healthcare in China: how a coordinated response by professional organizations helped older adults during an unprecedented crisis. International Psychogeriatrics, $\left[\begin{array}{lllll}s . & l .\end{array}\right], 9$ abr. DOI 10.1017/S1041610220000551. Disponível em: https://www.ncbi.nlm.nih.gov/pmc/articles/PMC7184143/. Acesso em: 11 maio 2020.

World Health Organization (2020). Supporting older people during the COVID-19 pandemic is everyone's business. EUROPE ed. Disponível em: http://www.euro.who.int/en/healthtopics/health-emergencies/coronavirus-covid-19/news/news/2020/4/supporting-older-peopleduring-the-covid-19-pandemic-is-everyones-business. Acesso em: 19 maio 2020.

\section{How to cite this article (APA format):}

Caldas, Luiza Thayline Vieira; Alcântara, João Victor da Silva; Arraes, Paula Pably Batista; Dias, Priscila Teixeira; Oliveira, Ramille Gonçalves; Rolim Neto, Modesto Leite; Nobre, Camila Bezerra (2020). Mental Health of the Elderly Population amid Pandemic COVID-19. Am. In. Mult. J., Jul to Oct. (9) 5, 57-62.

Received: 06/18/2020

Accepted: 07/29/2020 